\title{
ROUGHNESS OF TWO NORMS ON MUSIELAK-ORLICZ FUNCTION SPACES
}

\author{
JIMIN ZHENG \\ College of Computer Science and Technology \\ Chongqing University of Posts and Telecommunications \\ 400065 Chongqing, China \\ LIHUAN SUN \\ Department of Mathematics and Physics \\ AnHui University of Science and Technology \\ 232001 HuaiNan, AnHui, China \\ YUN'AN CUI \\ Department of Mathematics \\ Harbin University of Science and Technology \\ 150080 Harbin, HeiLongjiang, China \\ E-mail: cui@hotmail.com
}

\begin{abstract}
Some criteria of strong roughness, roughness and pointwise roughness of Orlicz norm and Luxemburg norm on Musielak-Orlicz function spaces are obtained.
\end{abstract}

1. Introduction. Leach and Whitefield [1] introduced the concept of rough norm in 1973. Later John and Zizler [2] and $\mathrm{Li}$ [3] introduced the concept of strong rough norm and pointwise rough norm, respectively. $X$ denotes a Banach space, $X^{*}$ the dual of $X$. $S(X)$ is the unit sphere in $X . B(X)$ denotes the unit ball of $X$. The norm of $X$ is said to be pointwise rough provided $\varepsilon(x)>0$ for every point on $S(X)$, where

$$
\begin{aligned}
\varepsilon(x)=\sup \left\{\varepsilon>0: \exists f_{n}, g_{n} \in X^{*},\left\|f_{n}\right\|,\left\|g_{n}\right\| \rightarrow\right. & 1, \\
& \left.f_{n}(x), g_{n}(x) \rightarrow 1, \varlimsup_{n \rightarrow \infty}\left\|f_{n}-g_{n}\right\| \geq \varepsilon\right\} .
\end{aligned}
$$

2000 Mathematics Subject Classification: 46B20, 46E30.

Key words and phrases: Musielak-Orlicz function space, rough norm, extreme point.

Supported by NSF of China, grant no. 10571037.

The paper is in final form and no version of it will be published elsewhere. 
$\|\cdot\|$ is said to be rough provided $\inf \{\varepsilon(x): x \in S(X)\}>0,\|\cdot\|$ is said to be strongly rough provided $\inf \{\operatorname{diamA}(\mathrm{x}): \mathrm{x} \in \mathrm{S}(\mathrm{x})\}>0$, where $A(x)=\left\{f \in S\left(X^{*}\right): f(x)=\|x\|=1\right\}$, that is, $A(x)$ is the gradient of $x$.

Criteria for roughness of the norm in Orlicz spaces are given in [10, 11]. This paper gives criteria for roughness of the norms in Musielak-Orlicz function spaces.

The triple $(T, \Sigma, \mu)$ stands for a finite nonatomic measure space. A mapping $T \times$ $[0, \infty) \rightarrow[0, \infty]$ is said to be a Musielak-Orlicz function if it satisfies:

(i) $M(\cdot, u)$ is $\Sigma$-measurable for any $u \in[0, \infty)$.

(ii) for $\mu$-a.e. $t \in T, M(t, u)$ is a left continuous and convex function of $u$.

(iii) for $\mu$-a.e. $t \in T, M(t, 0)=0$, $\lim _{u \rightarrow \infty} M(t, u)=\infty$, and there exists $u^{\prime} \neq 0$ such that $M\left(t, u^{\prime}\right)<\infty$ for $\mu$-a.e $t \in T$.

The complementary function of $M$ is defined by

$$
N(t, v)=\sup \{u v-M(t, u)\} \quad(\mu \text {-a.e. } t \in T ; v \geq 0)
$$

It is easy to see that $N(t, v)$ is also a Musielak-Orlicz function. We define $\hat{a}(t)=$ $\sup \{v \geq 0: N(t, v)<\infty\}$.

$p(t, u)$ and $p^{-}(t, u)$ (resp. $q(t, v)$ and $\left.q^{-}(t, v)\right)$ stand for the right and left derivative of $M(t, u)(N(t, v)$, respectively). We know that for any $u, v \geq 0$

$$
u v \leq M(t, u)+N(t, v) \quad(\mu \text {-a.e. } t \in T)
$$

and $u v=M(t, u)+N(t, v)$ if and only if $p^{-}(t, u) \leq v \leq p(t, u)$ or $q^{-}(t, v) \leq u \leq q(t, v)$.

$M$ is said to satisfy the $\Delta_{2}$ condition (for short, $M \in \Delta_{2}$ ) if there exist $\lambda>1$ and a measurable nonnegative function $\delta$ defined on $T$ such that $\int_{T} \delta(t) d \mu<\infty$ and

$$
M(t, 2 u) \leq \lambda M(t, u)+\delta(t) \quad(\text { a.e. } t \in T,-\infty<u<+\infty) .
$$

By $L^{0}$ we denote the set of all (equivalence classes of) $\Sigma$-measurable real functions defined on $T$. The linear set

$$
\left\{x(t) \in L^{0}: \varrho_{M}(\lambda x)=\int_{T} M(t, \lambda x(t)) d \mu \text { for some } \lambda>0\right\}
$$

endowed with the Luxemburg norm

$$
\|x\|_{M}=\inf \left\{\lambda>0: \rho_{M}(x / \lambda) \leq 1\right\}
$$

or the Orlicz norm

$$
\|x\|_{M}^{o}=\sup \left\{\int_{T} x(t) y(t) d \mu: \rho_{N}(y) \leq 1\right\}=\inf _{k>0}\left\{\frac{1}{k}\left(1+\rho_{M}(k x)\right)\right\}
$$

is a Banach space. We call it the Musielak-Orlicz space and denote by $L_{M}$ or $L_{M}^{o}$, respectively.

We define a closed subspace $E_{M}^{o}$ of $L_{M}^{o}$ by

$$
E_{M}^{o}=\left\{x \in L^{0}: \rho_{M}(\lambda x)<\infty \text { for any } \lambda>0\right\} .
$$

The spaces $E_{M}^{o}$ and $L_{M}^{o}$ coincide if and only if $M \in \Delta_{2}$.

For any $x \in L_{M}^{o}$, write

$$
\begin{gathered}
d^{o}(x)=\inf \left\{\|x-y\|_{M}^{0}: y \in E_{M}^{o}\right\}, d(x)=\inf \left\{\|x-y\|_{M}: y \in E_{M}\right\}, \\
\theta_{M}(x)=\inf \left\{\lambda>0: \rho_{M}(x / \lambda)<\infty\right\}=\xi(x) .
\end{gathered}
$$

It is known that $\theta_{M}(x)=d^{o}(x)=d(x)$. 
If $\int_{T} N(t, \hat{a}(t)) d \mu>1$, we have $\left[k_{x}^{*}, k_{x}^{* *}\right] \neq \emptyset$ and we know (see [13], [14]) that $\|x\|_{M}^{o}=$ $\frac{1}{k}\left(1+\rho_{M}(k x)\right)$ if and only if $k \in\left[k_{x}^{*}, k_{x}^{* *}\right]$, where

$$
\begin{aligned}
k_{x}^{*} & =\inf \left\{k>0: \int_{T} N(t, p(t, k|x(t)|)) d \mu \geq 1\right\}, \\
k_{x}^{* *} & =\sup \left\{k>0: \int_{T} N(t, p(t, k|x(t)|)) d \mu \leq 1\right\} .
\end{aligned}
$$

The dual space of $L_{M}$ is represented as $\left(L_{M}\right)^{*}=L_{N}^{o} \oplus \Phi$, i.e. every $f \in\left(L_{M}\right)^{*}$ is uniquely represented in the form $f=y+\phi$, where $\phi$ is a singular functional, i.e. $\phi(x)=0$ for any $x \in E_{M}$, and $y$ is a regular functional defined by the formula

$$
\langle x, y\rangle=\int_{T} x(t) y(t) d \mu \quad\left(\forall x \in L_{M}\right) .
$$

If any $f \in\left(L_{M}\right)^{*}$ is uniquely represented in the form $f=y+\phi$, then $\|f\|^{o}=$ $\|y\|_{N}^{o}+\|\phi\|^{o}$ (see [4, Lemma 1.3]), where

$$
\|\phi\|=\|\phi\|^{o}=\sup \left\{\phi(x): \rho_{M}(x)<\infty\right\}=\sup \left\{\phi(x): \rho_{M}(x)<\varepsilon\right\}=\sup _{\theta_{M}(x) \neq 0} \frac{\phi(x)}{\theta_{M}(x)}
$$

if the norm $\|f\|^{0}$ is defined by $\|f\|^{0}=\sup \left\{x^{*}(x):\|x\|_{M} \leq 1\right\}$.

If $f \in\left(L_{M}^{o}\right)^{*}$, that is, $f=y+\phi$, where $y \in\left(L_{N}\right)$ is the regular functional defined by the formula

$$
\langle x, y\rangle=\int_{T} x(t) y(t) d \mu \quad\left(\forall x \in L_{M}^{o}\right)
$$

and $\phi$ is a singular functional, then

$$
\|f\|=\inf \left\{\xi>0: \rho_{N}(y / \xi)+\|\phi\| / \xi \leq 1\right\}
$$

(see [14, Lemma 1.4]), if the norm $\|f\|$ is defined by the formula

$$
\|f\|=\sup \left\{x^{*}(x):\|x\|_{M}^{0} \leq 1\right\} .
$$

We start with auxiliary lemmas.

LEMMA 1. The spaces $E_{M}^{o}$ and $E_{M}$ are separable.

Lemma 2. (i) The spaces $E_{M}^{o}$ and $E_{M}$ are weakly Asplund.

(ii) The spaces $E_{M}^{o}$ and $E_{M}$ are Asplund if and only if $M \in \nabla_{2}$.

Proof. By Lemma 1, the proof is similar to that for Orlicz spaces (see [13], Theorem 2.58).

Lemma 3 ([6], Proposition 5.6). For any sequence $\left(u_{n}\right)$ in $L_{M}$ we have that $\rho_{M}\left(u_{n}\right) \rightarrow 1$ if and only if $\left\|u_{n}\right\|_{M} \rightarrow 1$ if and only if $M \in \Delta_{2}$.

LEMma 4 ([8], Lemma 1.7). There is no nonzero singular functional $\phi \in\left(L_{M}^{o}\right)^{*}$ attaining its norm on $S\left(L_{M}^{o}\right)^{*}$.

Lemma 5. For any $f \in\left(L_{M}^{o}\right)^{*}, f=y+\phi$ (where $y \in\left(L_{N}\right)$, $\phi$ is a singular functional). If $\|f\|=1$ is attained on $S\left(L_{M}^{o}\right)$, we obtain

$$
\int_{T} N(t, y(t)) d \mu+\|\phi\|=1 .
$$


Proof. Since Lemma 4 holds, we can get the result in the same way as for Orlicz spaces (see [6], Theorem 1.42).

LEMma 6. The following conditions are equivalent:

1) $N \in \Delta_{2}$, i.e., there exist $\lambda>1$ and $0 \leq \delta(t) \in L_{1}$ satisfying

$$
N(t, 2 v) \leq \lambda N(t, v)+\delta(t) \quad(\text { a.e. } t \in T, v \in \mathbf{R}) .
$$

2) for any $\varepsilon>0$, there exist $\lambda>1$ and $0 \leq \delta(t) \in L_{1}$, such that

$$
N(t, v / \varepsilon) \leq \lambda N(t, v)+\delta(t) \quad(\text { a.e. } t \in T, v \in \mathbf{R}) .
$$

3) for any $\varepsilon \in(0,1)$, there exist $\theta \in(0,1)$ and $0 \leq \delta(t) \in L_{1}$ satisfying

$$
M(t, \varepsilon u) \leq \theta \varepsilon M(t, u)+\delta(t) \quad(\text { a.e. } t \in T, u \in \mathbf{R}) .
$$

4) there exist $\varepsilon, \theta \in(0,1)$ and $0 \leq \delta(t) \in L_{1}$ satisfying:

$$
M(t, \varepsilon u) \leq \theta \varepsilon M(t, u)+\delta(t) \quad(\text { a.e. } t \in T, u \in \mathbf{R}) .
$$

Proof. See [13].

LEMMA 7 (see [19]). If

$$
K_{M}:=\sup _{\|x\|_{M}^{o}=1}\left\{k>0:\|x\|_{M}^{o}=\frac{1}{k}\left(1+\rho_{M}(k x)\right)\right\},
$$

then $K_{M}<\infty$ if and only if $N \in \Delta_{2}$.

Proof. Necessity. Since $N \notin \Delta_{2}$, taking any $\varepsilon>0$, putting

$$
\delta(t)=\sup \left\{v \geq 0: M(t, \varepsilon v)>\frac{\varepsilon}{\varepsilon+1} M(t, v)\right\}
$$

we have $\int_{T} M(t, \delta(t)) d t=\infty$. Indeed otherwise

$$
M(t, \varepsilon v(t)) \leq \frac{\varepsilon}{\varepsilon+1}(M(t, v)+M(t, \delta(t))) \quad(\text { a.e. } t \in T, v \in \mathbf{R}),
$$

whence, by Lemma 6 , we get $N \in \Delta_{2}$, a contradiction.

In this way, we get $u(t) \geq 0$ such that

$$
M(t, \varepsilon v(t))>\frac{\varepsilon}{\varepsilon+1} M(t, v(t)) \text { and } \int_{T} M(t, v(t)) d t>\frac{\varepsilon+1}{\varepsilon} .
$$

Then $\int_{T} M(t, \varepsilon v(t)) d t>1$, whence $\|\varepsilon u\|^{o}>1$. Therefore, there exists $\Omega \subset T$ such that $\left\|\left.\varepsilon u\right|_{\Omega}\right\|^{o}=1$. Take $k \in K\left(\left.\varepsilon u\right|_{\Omega}\right)$, i.e.

$$
1=\|\varepsilon u\|^{o}=\frac{1}{k}\left(1+\rho_{M}(k \varepsilon u)\right) .
$$

We have $k>1$, so

$$
\begin{aligned}
\frac{1}{k}+\rho_{M}\left(\left.k \varepsilon u\right|_{\Omega}\right) & \leq \frac{1}{k}\left(1+\rho_{M}\left(\left.k \varepsilon u\right|_{\Omega}\right)\right)=\left\|\left.\varepsilon u\right|_{\Omega}\right\|^{o} \\
& \leq \varepsilon\left(1+\rho_{M}\left(\left.\frac{1}{\varepsilon} \cdot \varepsilon u\right|_{\Omega}\right)\right)=\varepsilon\left(1+\rho_{M}\left(\left.v\right|_{\Omega}\right)\right) \\
& \leq \varepsilon\left(1+\frac{\varepsilon+1}{\varepsilon} \int_{\Omega} M(t, \varepsilon u(t)) d \mu\right)=\varepsilon+(1+\varepsilon) \rho_{M}\left(\left.\varepsilon u\right|_{\Omega}\right) .
\end{aligned}
$$


This shows that

$$
\frac{1}{k} \leq \varepsilon+\varepsilon \rho_{M}\left(\left.\varepsilon u\right|_{\Omega}\right) \leq 2 \varepsilon
$$

By the arbitrariness of $\varepsilon>0$, we obtain that $K_{M}=\infty$, a contradiction.

Sufficiency. Because $N \in \Delta_{2}$, by Lemma 6, there exist $\eta>0$ and $0<\delta(t) \in L_{1}$ such that

$$
M(t, 2 u) \geq 2(1+2 \eta) M(t, u)-\delta(t) \quad(\text { a.e. } t \in T, u \in \mathbb{R}) .
$$

Hence, for $u \geq 0$ satisfying $M(t, u)>\delta(t) / 2 \eta$, we obtain

$$
M(t, 2 u) \geq 2(1+\eta) M(t, u) .
$$

Pick $D$ big enough such that

$$
D-1-\frac{1}{2 \eta} \int_{T} \delta(t) \geq 1
$$

For any $x \in S\left(L_{M}^{o}\right)$, denote

$$
H_{x}=\{t \in T: M(t, D|x(t)|)>\delta(t) / 2 \eta\} .
$$

In view of $1=\|x\|_{M}^{o} \leq \frac{1}{D}\left(1+\rho_{M}(D x)\right)$, we get $\rho_{M}(D x) \geq D-1$. Moreover

$$
\begin{aligned}
\int_{H_{x}} M(t, D x(t)) d \mu & =\rho_{M}(D x)-\int_{T \backslash H_{x}} M(t, D x(t)) d \mu \\
& \geq D-1-\frac{1}{2 \eta} \int_{T \backslash H_{x}} \delta(t) d \mu \geq D-1-\frac{1}{2 \eta} \int_{T} \delta(t) d \mu \geq 1 .
\end{aligned}
$$

Since $N \in \Delta_{2}$, we have $\hat{a}(t)=\infty$ a.e. For any $x \in S\left(L_{M}^{o}\right)$, we obtain that $K(x) \neq \emptyset$. If $k \in K(x)$, then $k \leq D$ or $k>D$. If $k>D$, there exists $j \geq 1$ such that $2^{j-1} D<k \leq 2^{j} D$. From (1) and (2), we have

$$
\begin{aligned}
2^{j} D \geq k & =1+\rho_{M}(k x)>\int_{H_{x}} M(t, k x(t)) d \mu \geq \int_{H_{x}} M\left(t, 2^{j-1} D|x(t)|\right) d \mu \\
& \geq 2^{j-1}(1+\eta)^{j-1} \int_{H_{x}} M(t, D|x(t)|) d \mu \geq(1+\eta)^{j-1} 2^{j-1} .
\end{aligned}
$$

This shows that $j-1 \leq \log _{1+\eta}^{2} D$. Therefore $k \leq D \cdot 2^{\log _{1+\eta}^{2} D+1}$, so $K_{M}<\infty$.

LEMMA 8. The space $E_{M}$ is smooth if and only if $p(t, u)$ is continuous with respect to $u$, $t \in T$. The support functional of $u \in E_{M}$ is the function

$$
v(t)=\frac{p\left(t, u(t) /\|u\|_{M}\right) \operatorname{sign} u(t)}{\left\|p\left(t, u(t) /\|u\|_{M}\right)\right\|^{o}} \quad \mu \text {-a.e. }
$$

Proof. It is analogous to the proof of Theorem 2.15 in [6].

LEMMA 9 ([8], Lemma 1.9). If $x \in\left(L_{M}^{o}\right)$ and $\theta_{M}(x)>0$, there exist two distinct singular functionals $\phi_{i},\left\|\phi_{i}\right\|=1$, satisfying $\phi_{i}(u)=\xi_{0}(u), i=1,2$.

LEMMA 10. If $p(t, u)$ is continuous with respect to $u, t \in T$, then $\mu$-a.e. $u \in S\left(L_{M}\right)$ is a smooth point of $B\left(L_{M}\right)$ if and only if the support functional of $u$ belongs to $S\left(L_{N}^{o}\right)$, and $u \neq \theta$.

Proof. By Lemmas 8, 9, the proof is similar to the proof for Orlicz spaces (see [6], Theorem $2.17)$. 
LEMMA 11. $u \in S\left(L_{M}\right)$ is a smooth point of $B\left(L_{M}\right)$ if and only if

(1) $|u(t)|<B(t) \quad($ a.e. $t \in T)$;

(2) $\rho_{M}(u)=1, p^{-}(|u|) \in E_{N}^{o}$;

(3) $p^{-}(t,|u(t)|)=p(t,|u(t)|)$, where $B(T)=\sup \{u \geq 0: M(t, u)<\infty\}$.

Proof. Since Lemma 10 holds, we can repeat the proof from [16].

LEMMA 12 ([17]). $x \in S\left(L_{M}^{o}\right)$ is an extreme point of $B\left(L_{M}^{o}\right)$ if and only if

(a) the set $K(x)$ consists of one element from $(0,+\infty)$,

(b) $k x(t) \in S_{M}$ for $\mu$-a.e. $t \in T$, where $\{k\}=K(x)$.

LEMMA 13. $x_{0} \in S(X)$ is a smooth point if and only if its support functional is an extreme point of $B\left(X^{*}\right)$.

Lemma 14 (see [18]). If $M \in \Delta_{2}$ and $x_{0} \in S\left(L_{M}\right)$ is an extreme point of $B\left(L_{M}^{o}\right)$, then $x_{0}$ is an $H$-point.

\section{The Orlicz norm}

THEOREM 2.1. The following conditions are equivalent:

(i) $L_{M}^{o}$ is rough.

(ii) $L_{M}^{o}$ is pointwise rough.

(iii) $M \notin \nabla_{2}$.

Proof. (i) $\Rightarrow$ (ii) is trivial.

(ii) $\Rightarrow$ (iii) If $M \in \nabla_{2}$, by Lemma 2(ii), we obtain that $E_{M}^{o}$ is an Asplund space. By Corollary 2 in [12], p. 177, there is at least one F-differential point $x_{0}$ of $E_{M}^{o}$ on $S\left(E_{M}^{o}\right)$. Now, we need only prove that $x_{0}$ is also an F-differential point of $L_{M}^{o}$.

Let $f_{n} \in\left(L_{M}^{o}\right)^{*}, f_{n}=y_{n}+\phi_{n},\left\|f_{n}\right\| \rightarrow 1$ and $f_{n}\left(x_{0}\right) \rightarrow 1$, where $y_{n} \in L_{N}$ and $\phi_{n}$ is a singular functional,

$$
f_{n}(x)=\int_{T} x(t) y_{n}(t) d \mu+\phi_{n}(x) \quad\left(x \in L_{M}^{o}\right) .
$$

Since $\int_{T} x_{0}(t) y_{n}(t) d \mu=\int_{T} x_{0}(t) y_{n}(t) d \mu+\phi_{n}\left(x_{0}\right)=f_{n}\left(x_{0}\right), \underline{\lim }_{n \rightarrow \infty}\left\|y_{n}\right\|_{N} \geq 1$. If $\varlimsup_{n \rightarrow \infty}\left\|y_{n}\right\|_{N}>1$, there exists $\lambda>0$ such that $\left\|y_{n}\right\|_{N}>1$ for an infinite number of $n$. Therefore, $\left\|\frac{y_{n}}{1+\lambda}\right\|>1$ and $\rho_{N}\left(\frac{y_{n}}{1+\lambda}\right) \geq 1$. We have

$$
\left\|f_{n}\right\|=\inf \left\{\xi>0: \rho_{N}\left(y_{n} / \xi\right)+\phi_{n} / \xi \leq 1\right\} .
$$

It is easy to prove that $\left\|f_{n}\right\| \geq 1+\lambda$. This contradiction proves that

$$
\varlimsup_{n \rightarrow \infty}\left\|y_{n}\right\|_{N} \leq 1
$$

whence $\left\|y_{n}\right\|_{N} \rightarrow 1$. By $\lim _{n \rightarrow \infty}\left\|f_{n}\right\|=1$ and (2.1), there exists $\xi_{n} \rightarrow 1$ satisfying

$$
\rho_{N}\left(y_{n} / \xi_{n}\right)+\left\|\phi_{n}\right\| / \xi_{n} \leq 1 \text {. }
$$

Since $N \in \Delta_{2}$ and $\left\|y_{n}\right\|_{N} \rightarrow 1$, by Lemma 3 , we get $\rho_{N}\left(y_{n} / \xi_{n}\right) \rightarrow 1$, so $\left\|\phi_{n}\right\| / \xi_{n} \rightarrow 0$, i.e, $\left\|\phi_{n}\right\| \rightarrow 0$. Since $x_{0}$ is an F-differential point of $E_{M}^{o}$, and $\left\|y_{n}\right\|_{N} \rightarrow 1$, so $\left\{y_{n}\right\}$ is a Cauchy sequence. Combining this fact with $\left\|\phi_{n}\right\| \rightarrow 0$, we get that $\left\{f_{n}\right\}$ is a Cauchy sequence, too. Hence $x_{0}$ is an F-differential point of $L_{M}^{o}$. 
(iii) $\Rightarrow$ (i) Suppose that $x_{0} \in S\left(L_{M}^{o}\right), f=y+\phi \in\left(L_{M}^{o}\right)^{*},\|f\|=1$ and $f\left(x_{0}\right)=\|x\|_{M}^{o}$ $=1$. By Lemma 5,

$$
\int_{T} N(t, y(t)) d \mu+\|\phi\|=1 \quad \text { for a.e. } t \in T,
$$

Since $N \notin \Delta_{2}$, there exists $v \in S\left(L_{N}\right)$ and $\varepsilon_{0}>0$ satisfying

$$
\left\|v \chi_{T_{i}}\right\| \geq \varepsilon_{0}, \quad i=1,2, \ldots,
$$

where $T_{i}=\{t \in T:|v(t)| \geq i\}$.

Defining $v_{i}=v \chi_{T \backslash T_{i}} /\left\|v \chi_{T \backslash T_{i}}\right\|$, we have $\left\|v_{i}\right\|=1$. Define $f_{i}, g_{i} \in\left(L_{M}^{o}\right)^{*}$ by

$$
\begin{aligned}
& f_{i}(x)=\int_{T \backslash T_{i}} x(t) y(t) d \mu+\int_{T_{i}} v_{i} x(t) d \mu+\phi(x), \\
& g_{i}(x)=\int_{T \backslash T_{i}} x(t) y(t) d \mu-\int_{T_{i}} v_{i} x(t) d \mu+\phi(x) \quad\left(x \in L_{M}^{o}\right) .
\end{aligned}
$$

In view of

$$
\int_{T \backslash T_{i}} x_{0}(t) y(t) d \mu \rightarrow \int_{T} x_{0}(t) y(t) d \mu
$$

and

$$
\int_{T_{i}} v_{i} x(t) d \mu \leq \int_{T_{i}} M\left(t, x_{0}(t)\right) d \mu+\int_{T_{i}} N\left(t, v_{i}\right) d \mu \rightarrow 0,
$$

we get $\underline{\lim }_{i \rightarrow \infty} f_{i}\left(x_{0}\right)=1$, whence $\underline{\lim }_{i \rightarrow \infty}\left\|f_{i}\right\| \geq 1$. On the other hand, as $i \rightarrow \infty$, for any $\varepsilon>0$, there exists $T_{i_{o}}$ such that $\int_{T_{i_{o}}} N\left(t, v_{i_{o}}\right) d t<\varepsilon$, a.e. $t \in T$. By $(2.2)$, we know that

$$
\int_{T \backslash T_{i}} N(t, y(t)) d t+\int_{T_{i}} N\left(t, v_{i}(t)\right) d t+\|\phi\| \leq \rho_{N}(y)+\|\phi\|+\varepsilon=1+\varepsilon, \quad i \geq i_{0} .
$$

Taking into account (2.1), $\left\|f_{i}\right\| \leq 1+\varepsilon$. Therefore $\lim _{i \rightarrow \infty}\left\|f_{i}\right\|=1$.

Similarly, $\lim _{i \rightarrow \infty} g_{i}\left(x_{0}\right)=1, \lim _{i \rightarrow \infty}\left\|g_{i}\right\|=1$. But we have proved that $\lim _{i \rightarrow \infty}\left\|v_{i} \chi_{T_{i}}\right\|$ $=1$, hence $\overline{\lim }_{i \rightarrow \infty}\left\|f_{i}-g_{i}\right\|=2$. This shows that $\varepsilon\left(x_{0}\right) \geq 2$. In view of the arbitrariness of $x_{0}$, we get that $\inf \left\{\varepsilon(x): x \in S\left(L_{M}^{o}\right)\right\}=2$, and $L_{M}^{o}$ is rough.

THEOREM 2.2. The space $L_{M}^{o}$ is not strongly rough.

Proof. By Lemma 1, Lemma 2(i) and Lemma 5, the proof is the same as for Orlicz spaces, see $[10]$.

\section{The Luxemburg norm}

THEOREM 3.1. The space $L_{M}$ is pointwise rough if and only if $M \notin \nabla_{2}$.

Proof. Sufficiency. A non-smooth point must be a rough point. Next we prove that every smooth point is a rough point.

Suppose that $x_{0} \in S\left(L_{M}\right)$ is a smooth point. By Lemma 10, there exists a support functional of $x_{0}-y_{0}$, which belongs to $S\left(L_{N}^{o}\right)$. By the proof of Lemma 11, we know that $K\left(y_{0}\right) \neq \emptyset$. Choose $k_{0}>0$ satisfying $\frac{1}{k_{0}}\left(1+\rho_{N}\left(k_{0} y_{0}\right)\right)=\left\|y_{0}\right\|_{N}^{o}=1$. Since $N \notin \Delta_{2}$, we get from the proof of Theorem 2.1 that $v_{i}=v \chi_{T \backslash T_{i}} /\left\|v \chi_{T \backslash T_{i}}\right\|$, where $T_{i}=\{t \in T:|v(t)| \geq i\}$ 
satisfies $\left\|v_{i}\right\|=1$. Therefore, as $i \rightarrow \infty$, for any $\varepsilon>0$, there exists $T_{i_{o}}$ such that $\int_{T_{i_{o}}} N\left(t, v_{i_{o}}\right) d t<\varepsilon$ for a.e. $t \in T$ and $i_{o}>i$. Define $y_{i}, z_{i} \in L_{N}^{o}$ by

$$
y_{i}(t)=\left\{\begin{array}{ll}
y_{0}(t), & t \in T \backslash T_{i}, \\
v_{i} / k_{0}, & t \in T_{i},
\end{array} \quad z_{i}(t)= \begin{cases}y_{0}(t), & t \in T \backslash T_{i}, \\
-v_{i} / k_{0}, & t \in T_{i} .\end{cases}\right.
$$

Then

$$
\int_{T \backslash T_{i}} x_{0}(t) y_{0}(t) d \mu+\frac{v_{i}}{k_{0}} \int_{T_{i}} x_{0}(t) d t \rightarrow \int_{T} x_{0}(t) y_{0}(t) d \mu=1 .
$$

Hence $\underline{\lim }_{i \rightarrow \infty}\left\|y_{i}\right\|_{N}^{o} \geq 1$. Moreover,

$$
\begin{aligned}
\left\|y_{i}\right\|_{N}^{o} & \leq \frac{1}{k_{0}}\left(1+\rho_{N}\left(k_{0} y_{i}\right)\right)=\frac{1}{k_{0}}\left(1+\int_{T \backslash T_{i}} N\left(t, k_{0} y_{0}(t)\right) d t+\int_{T_{i}} N\left(t, v_{i}\right) d t\right) \\
& \leq \frac{1}{k_{0}}\left(1+\rho_{N}\left(k_{0} y_{i}\right)\right)+\varepsilon \frac{1}{k_{0}}=1+\varepsilon \frac{1}{k_{0}} .
\end{aligned}
$$

Therefore, $\varlimsup_{n \rightarrow \infty}\left\|y_{i}\right\|_{N}^{o} \leq 1$, whence $\lim _{i \rightarrow \infty}\left\|y_{i}\right\|_{N}^{o}=1$.

In the same way one can prove that $\int_{T} x_{0}(t) z_{i}(t) d \mu \rightarrow 1,\left\|z_{i}\right\|_{N}^{o} \rightarrow 1$. But

$$
\left\|y_{i}-z_{i}\right\|_{N}^{o} \geq\left\|y_{i}-z_{i}\right\|_{N} \geq \frac{2}{k_{0}}\left\|v_{i}\right\|_{N} \text {. }
$$

Hence $\varlimsup_{n \rightarrow \infty}\left\|y_{i}-z_{i}\right\|_{N}^{o} \geq 2 / k_{0}$, which shows that $x_{0}$ is a rough point of $L_{M}$.

Necessity. If $M \in \nabla_{2}$, by Lemma 2(ii), $E_{M}$ is an Asplund space. Moreover, $\left\|f_{n}\right\|_{M}^{o}=$ $\left\|y_{n}\right\|_{M}^{o}+\left\|\phi_{n}\right\|$, whence the proof is similar to the proof for Orlicz spaces.

THEOREM 3.2. The space $L_{M}$ is not strongly rough.

Proof. Since Lemma 1 and Lemma 2 (1) hold, the proof is as in the case of Orlicz spaces, see $[10]$.

THEOREM 3.3. The space $L_{M}$ is rough if and only if $M \notin \nabla_{2}$ and $M \in \Delta_{2}$.

Proof. Sufficiency. Since $M \in \Delta_{2}$, the support functional at any point $x_{0}$ on $S\left(L_{M}\right)$ belongs to $S\left(L_{N}\right)$. Since $M \notin \nabla_{2}$, by the proof of the sufficiency in Theorem 2.1, $\varepsilon\left(x_{0}\right) \geq$ $2 / k_{y}$, where $\|y\|_{N}^{o}=1, \int_{T} x_{0}(t) y(t) d \mu=1$. Again by Lemma 7 , we have $\sup \left\{k_{y}:\|y\|_{N}^{o}=1\right\}$ $=k<\infty$, whence $\inf \left\{\varepsilon(x): x \in S\left(L_{M}\right)\right\} \geq 2 / k$, which shows that $L_{M}$ is rough.

Necessity. Roughness implies pointwise roughness, so it follows immediately from Theorem 2.1 that $M \notin \nabla_{2}$.

In order to prove that $M \in \Delta_{2}$, we consider the following four steps.

(I) If $M \notin \Delta_{2}$, for any positive integer $n$, there exists a smooth point $x_{0}$ on $S\left(L_{M}\right)$. From the proof of Lemma 11, we get $y_{0}$ in the support of $x_{0}$ satisfying $K\left(y_{0}\right) \neq \emptyset$, moreover we prove that $k_{y_{o}}>n$.

Assume that $M \notin \Delta_{2}$, take any $\varepsilon>0(\varepsilon=1 / 2 n)$ and put

$$
\delta(t)=\sup \left\{v \geq 0: N(t, \varepsilon v)>\frac{\varepsilon}{\varepsilon+1} N(t, v)\right\} .
$$

Then $\int_{T} N(t, \delta(t)) d t=\infty$. Indeed, otherwise

$$
N(t, \varepsilon v(t)) \leq \frac{\varepsilon}{\varepsilon+1}(N(t, v)+N(t, \delta(t))) \quad(\text { a.e. } t \in T, v \in \mathbf{R}),
$$

so by Lemma 6 , we get that $M \in \Delta_{2}$, a contradiction. 
In this way, we get $v(t) \geq 0$, satisfying: $v(t)$ is a strict increase point of $q(t, v)$ with respect to $v$ for each $t \in T$ and

$$
N(t, \varepsilon v(t))>\frac{\varepsilon}{\varepsilon+1} N(t, v(t)), \quad \int_{T} N(t, v(t)) d t>\frac{\varepsilon+1}{\varepsilon} .
$$

Therefore $\int_{T} N(t, \varepsilon v(t)) d t>1$, whence $\|\varepsilon v\|>1$. Moreover, $\|\varepsilon v\|^{o} \geq\|\varepsilon v\|$, so we obtain $\|\varepsilon v\|^{o}>1$. Consequently there exists $\Omega \subset T$ such that $\left\|\left.\varepsilon v\right|_{\Omega}\right\|^{o}=1$. Take $k \in K\left(\left.\varepsilon v\right|_{\Omega}\right)$, i.e.

$$
1=\|\varepsilon v\|^{o}=\frac{1}{k}\left(1+\rho_{N}(k)\right) .
$$

Then $k>1$ and

$$
\begin{aligned}
\frac{1}{k}+\rho_{N}\left(\left.k \varepsilon v\right|_{\Omega}\right) & \leq \frac{1}{k}\left(1+\rho_{N}\left(\left.k \varepsilon v\right|_{\Omega}\right)\right)=\left\|\left.\varepsilon v\right|_{\Omega}\right\|^{o} \\
& \leq \varepsilon\left(1+\rho_{N}\left(\left.\frac{1}{\varepsilon} \cdot \varepsilon v\right|_{\Omega}\right)\right)=\varepsilon\left(1+\rho_{N}\left(\left.v\right|_{\Omega}\right)\right) \\
& \leq \varepsilon\left(1+\frac{\varepsilon+1}{\varepsilon} \int_{\Omega} N(t, \varepsilon v(t)) d \mu\right)=\varepsilon+(1+\varepsilon) \rho_{N}\left(\left.\varepsilon v\right|_{\Omega}\right),
\end{aligned}
$$

which shows that $\frac{1}{k} \leq \varepsilon+\varepsilon \rho_{N}\left(\left.\varepsilon v\right|_{\Omega}\right) \leq 2 \varepsilon$, i.e. $\left.k \varepsilon v\right|_{\Omega}>n$.

Since $\int_{T} N(t, \delta(t))=\infty, \varepsilon=1 / 2 n$ and $v(t)<\delta(t)$, we get $\int_{T} N(t, \varepsilon v(t))<\infty$. Therefore $N(t, \varepsilon v(t))$ is bounded for $\mu$-a.e. $t \in T$. Hence $\varepsilon v(t)$ is bounded for $\mu$-a.e. $t \in T$. Therefore $\left.\varepsilon v(t)\right|_{\Omega} \in E_{N}^{0}$. By the Hahn-Banach Theorem, there is an $x_{0} \in S\left(L_{M}\right)$ such that $x_{0}\left(\left.\varepsilon v\right|_{\Omega}\right)=\left\|\left.\varepsilon v\right|_{\Omega}\right\|_{N}^{0}=1=\left\|x_{0}\right\|_{M}$. Therefore $\left.\varepsilon v\right|_{\Omega}$ is a support functional at $x_{0}$.

Setting $y_{0}=\left.\varepsilon v\right|_{\Omega}$, from the above proof we obtain that $k_{y_{0}}>n$.

(I) Since $\varepsilon v$ is a strict increase point of $q(t, v)$ with respect to $v$ for each $t \in T$, we know that $N(t, v)$ is strictly convex with respect to $v$ for each $t \in T, k y_{0}$ is a strictly convex point of $N(t, v)$. By Lemma 12, we obtain that $y_{0}$ is an extreme point of $L_{N}^{0}$. So by Lemma $13, x_{0}$ is a smooth point of $L_{M}$.

(III) Put $y_{n} \in L_{N}^{0},\left\|y_{n}\right\|_{N}^{0}=1$ and $\int_{T} x_{0}(t) y_{n}(t) d \mu \rightarrow 1$. Then $y_{n}(t)-y_{0}(t) \stackrel{\mu}{\longrightarrow} 0$. By the same method of Lemma 14 , we get $y_{n}(t)-y_{0}(t) \stackrel{\mu}{\longrightarrow} 0$ on $\Omega$.

Next we will prove that $y_{n}(t)-y_{0}(t) \stackrel{\mu}{\longrightarrow} 0$ on $t \in T \backslash \Omega$. Since $\int_{T} y_{n}(t) \chi_{\Omega} x_{0}(t) d \mu \rightarrow 1$, we have $\left\|y_{n}\right\|_{N}^{0} \rightarrow 1$. Notice that

$$
1 \leftarrow\left\|y_{n}\right\|_{N}^{0}=\frac{1}{k_{n}}\left(1+\rho_{N}\left(k_{n} y_{n} \chi_{T \backslash \Omega}\right)\right) \geq\left\|y_{n} \chi_{T \backslash \Omega}\right\|_{N}^{0}+\rho_{N}\left(y_{n} \chi_{T \backslash \Omega}\right) .
$$

Therefore $\rho_{N}\left(y_{n} \chi_{T \backslash \Omega}\right) \rightarrow 0$, whence $y_{n} \rightarrow 0$ on $t \in T \backslash \Omega$.

(IV) We have $\varlimsup_{n \rightarrow \infty}\left\|y_{n}-y_{0}\right\|_{N}^{0} \leq 4 / k$. For any $\varepsilon>0$, there exists $\delta>0$ such that $e \subset t, \mu(e)<\delta$ implies $\rho_{N}\left(k y_{0} \chi_{e}\right)<\varepsilon$ and $\left\|y_{0} \chi_{e}\right\|_{N}^{0}<\varepsilon$. Pick $e_{0} \subset t$ such that $\mu\left(e_{0}\right)<\delta$, and $y_{n}(t)$ converges to $y_{0}(t)$ uniformly on $T \backslash e_{0}$. Then for $n$ large enough, we have

$$
\begin{gathered}
1+\varepsilon \geq\left\|y_{n}\right\|_{N}^{0}=\frac{1}{k_{n}}\left(1+\rho_{N}\left(k_{n} y_{n} \chi_{T \backslash e_{0}}\right)+\rho_{N}\left(k_{n} y_{n} \chi_{e_{0}}\right)\right) \\
\geq\left\|y_{n} \chi_{T \backslash e_{0}}\right\|_{N}^{0}+\frac{\rho_{N}\left(k_{n} y_{n} \chi_{e_{0}}\right)}{2 k} \geq\left\|y_{n} \chi_{T \backslash e_{0}}\right\|_{N}^{0}-\varepsilon \\
+\frac{\rho_{N}\left(k_{n} y_{n} \chi_{e_{0}}\right)}{2 k} \geq 1-2 \varepsilon+\frac{\rho_{N}\left(k_{n} y_{n} \chi_{e_{0}}\right)}{2 k}
\end{gathered}
$$


whence $\rho_{N}\left(k_{n} y_{n} \chi_{e_{0}}\right)<\varepsilon$. Therefore, for $n$ large enough

$$
\begin{aligned}
\rho_{N}\left(\frac{k}{4}\left(y_{n}-y_{0}\right)\right) & \leq \rho_{N}\left(\frac{k}{4}\left(y_{n}-y_{0}\right) \chi_{T \backslash e_{0}}\right)+\frac{1}{2} \rho_{N}\left(\frac{k-k_{n}}{2} y_{n} \chi_{e}\right) \\
& +\frac{1}{4} \rho_{N}\left(k_{n} y_{n} \chi_{e_{0}}\right)+\frac{1}{4} \rho_{N}\left(k y_{0} \chi_{e_{0}}\right)=o(\varepsilon) .
\end{aligned}
$$

Hence

$$
\left\|y_{n}-y_{0}\right\|_{N}^{0} \leq \frac{4}{k}\left(1+\rho_{N}\left(\frac{k}{4}\left(y_{n}-y_{0}\right)\right)\right) \leq \frac{4}{k}+o(\varepsilon) .
$$

So $\lim _{n \rightarrow \infty}\left\|y_{n}-y_{0}\right\|_{N}^{0} \leq \frac{4}{k}$. This shows that $\varepsilon\left(x_{0}\right) \leq 8 / k \leq 8 / n$, whence $\inf \{\varepsilon(x): x \in$ $\left.S\left(L_{M}\right)\right\}=0$, i.e. $L_{M}$ is not rough.

\section{References}

[1] F. B. Leach and H. M. Whitfield, Differentiable functions and rough norms on Banach spaces, Proc. Amer. Math. Soc. 33 (1972), 120-126.

[2] K. John and Y. Zizler, On rough norms on Banach spaces, Comment. Math. Univ. Carolin. 19 (1978), 335-349.

[3] X. J. Li, The rough norms and non-differentiability of Banach spaces, Chin. Math. Ann. 8A (1987), 621-625.

[4] G. Godini, Rough and strongly rough norms on Banach spaces, Proc. Amer. Math. Soc. 87 (1983), 239-245.

[5] M. A. Krasnoselskii and Ya. B. Rutickii, Convex Functions and Orlicz Spaces, Noordhoff, Groningen, 1996.

[6] C. X. Wu, T. F. Wang, S. T. Chen and Y. W. Wang, Geometry of Orlicz Spaces, Harbin, 1986.

[7] H. Hudzik and Y. I. Ye, Support functionals and smoothness in Musielak-Orlicz sequence spaces endowed with the Luxemburg norm, Comment. Math. Univ. Carolin. 31 (1990), 661-684; correction, 35 (1994), 209.

[8] C. X. Wu and H. I. Sun, Norm calculation and complex convexity of Musielak-Orlicz sequence spaces, Chinese Ann. Math. 12A (1991), suppl., 98-102.

[9] Y. A. Cui, H. Hudzik and R. Płuciennik, Extreme points and strong extreme points in Orlicz spaces equipped with Orlicz norm, Z. Anal. Anwendungen 22 (2003), 789-817.

[10] Y. A. Cui and T. F. Wang, The roughness of the norms on Orlicz spaces, Comment. Math. (Prace Mat.) 31 (1991), 49-57.

[11] Y. A. Cui and T. F. Wang, The roughness on Orlicz sequence spaces, Harbin Sci.Tech. 17 (1993), 70-75.

[12] J. Diestel, Geometry of Banach Spaces, Springer-Verlag, 1975.

[13] S. T. Chen, Geometry of Orlicz spaces, Dissertationes Mathematicae 356 (1996).

[14] H. Hudzik and Z. Zbąszyniak, Smoothness in Musielak-Orlicz space equipped with the Orlicz norm, Collect. Math. 48 (1997), 543-561.

[15] T. F. Wang and S. R. Bian, Smooth points and strong (very) smooth points of MusielakOrlicz spaces with Orlicz norm, Acta Anal. Funct. Appl. (1999), 61-68.

[16] F. F. Yu and J. Li, $w^{*}$-smooth points of Musielak-Orlicz spaces, Acta Anal. Funct. Appl. 4 (2002), 185-192. 
[17] L. H. Sun and Y. A. Cui, Extreme points in a Musielak-Orlicz function space equipped with the Orlicz norm, Natur. Sci. J. Harbin Normal Univ. 22 (2006), 14-17 (in Chinese).

[18] L. H. Sun and Y. A. Cui, H-points in a Musielak-Orlicz function space equipped with the Orlicz norm, to appear.

[19] L. Y. Cao, Master Paper, 2003. 
